\title{
Development of the Meter for Measuring Pork Quality Based on the Electrical Characteristics*
}

\author{
Zhen Xing $^{1}$, Wengang Zheng ${ }^{2}$, Changjun Shen ${ }^{1,2}$, and Xin Zhang ${ }^{1,2}$ \\ ${ }^{1}$ Beijing Research Center for Intelligent Agricultural Equipment, 100097, Beijing, China \\ ${ }^{2}$ National Engineering Research Center for Information Technology in Agriculture, \\ 100097, Beijing, China \\ \{xingz, zhengwg, shencj, zhangx\} @nercita.org.cn
}

\begin{abstract}
The excitation frequency on the electrical characteristics of pork had been identified by the experiments of the electrical characteristics and the theory of electrical characteristics, the relationship between the evaluation of quality of pork and the impedance characteristics was established, and a nondestructive portable meter for measuring pork quality was developed. The functions of each hardware and software design of the meter were described in detail. The result shows that the conductivity of the pork increases with the level of corruption by the experiment of the measurement selected sample of pork. The pork quality meter based on the electrical characteristics provides the measurement method and apparatus of a low-cost, rapid, qualitative assessment of pork quality.
\end{abstract}

Keywords: the electrical characteristics, pork quality, a portable meter, a lowcost.

\section{Introduction}

Food safety become the focus of attention when people's living standard have been rising, The meat is the main food in china; China is one of the biggest countries for the production of livestock and poultry meat and its products. According to The People's Republic of China Statistical Yearbook 2008, China's pork production in 2007 was 42.878 million tons, the amount of production and consumption all ranks the world. People are cautious about meat consumption when meat safety incidents, such as, the injection meat or the clenbuterol, are frequently exposed [1]. In order to eliminate consumer concern for meat product, some approaches should be taken, such as, improving the moral quality of the farmers and producers, and preventing non-healthy meat to target into the market. So the measuring meter for fast detection of meat quality is an important role in the quarantine before meat onto the market.

* The paper was supported by the National High Technology Research and Development Program("863"Program) of China (2007AA10 Z212). 
Currently, there are a lot of pork quality detection methods. For example, the fat of the pig ketene body and the lean meat were graded by using spectroscopy or ultrasonic detection in the abroad; the nutritional composition in meat and meat quality changes are studied by using the equipment of near-infrared spectroscopy or the chemo metrics in the domestic; the study group of Ding[2] had been studied the nondestructive optical detection methods for the fatty tissue, and measured the content of the Hemoglobin and the myoglobin in different depths of muscle; the study group of Huang $[3,4]$ had been studied the optical parameter properties of agricultural products, the detection of fresh pork and deep water of the fresh pork. In addition, the meat quality of detection methods, based on dielectric properties or electrochemical properties, had been developed rapidly. For example, Zhang [5] had been studied temperature effect on the dielectric properties of the lunch pork roll; Kent [6] soaked the meat in water at different times, attained different the water content, and studied the water content effect on the dielectric properties of chicken, scallops and pork. Zhang [7] had been studied the salt effect on the permittivity and dielectric loss factor of the pork. Currently, the dielectric properties of the raw meat and meat products had been studied in abroad, especially the microwave dielectric properties, however, detecting the quality of pork by using the dielectric properties has only begun in the domestic.

A new sensor based on the transmission line impedance theory was designed by using the coaxial transmission line technology, and the meter for the meter for measuring pork quality based on this new sensor was developed in this paper; it can be more convenient, fast and efficient measurement of pork quality, and ensure the safety of the pork quality.

\section{Sensor Design}

The sensor consists of a power supply unit, a high-frequency signal source, a signal processing unit, a coaxial transmission line and measuring probes. The structure is as shown in Figure 1.

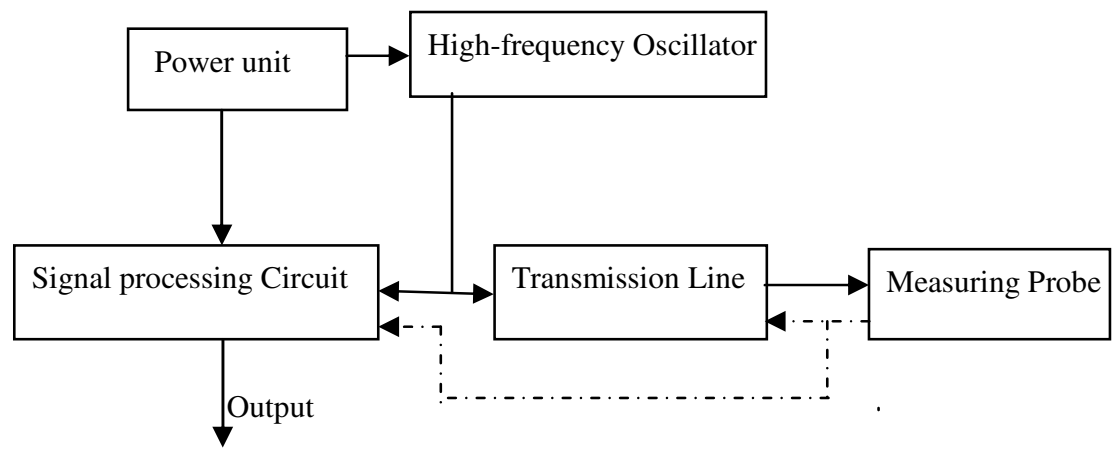

Fig. 1. The principle structure of the sensor 


\subsection{Measuring Principle}

Coaxial transmission line measurement technique is that the electrical characteristics of biological tissue (such as conductivity, dielectric constant, etc.) was transferred into high-frequency electromagnetic wave reflection coefficient, the relationship between the electrical characteristics of biological tissue and high frequency electromagnetic wave reflection coefficient, the electrical characteristics of biological tissue can be obtained by measuring the radiation coefficient. When the power supply was at work, a fixed-and high-frequency signal, generated by the crystal oscillator, transmitted through the coaxial transmission line to the probe which was inserted into the testing material. Due to the impedance's mismatching, parts of the signals would return and superpose with the latter input ones. Consequently, it set up a doable way of associating a stabilized amplitude voltage with the coaxial transmission line and the probe, which is available to the quality detecting.

\subsection{Signal Processing Circuit}

According to signal detection theory, the signal processing circuit of accuracy, stability, sensitivity directly determines the overall system performance, therefore, the design of the signal processing circuit is crucial, it must eliminate noise and amplify useful signal. Aiming at catering to this need, the measurement circuit can be simplified as two-stage amplification. The signal processing circuit is shown in detail in Figure 2.

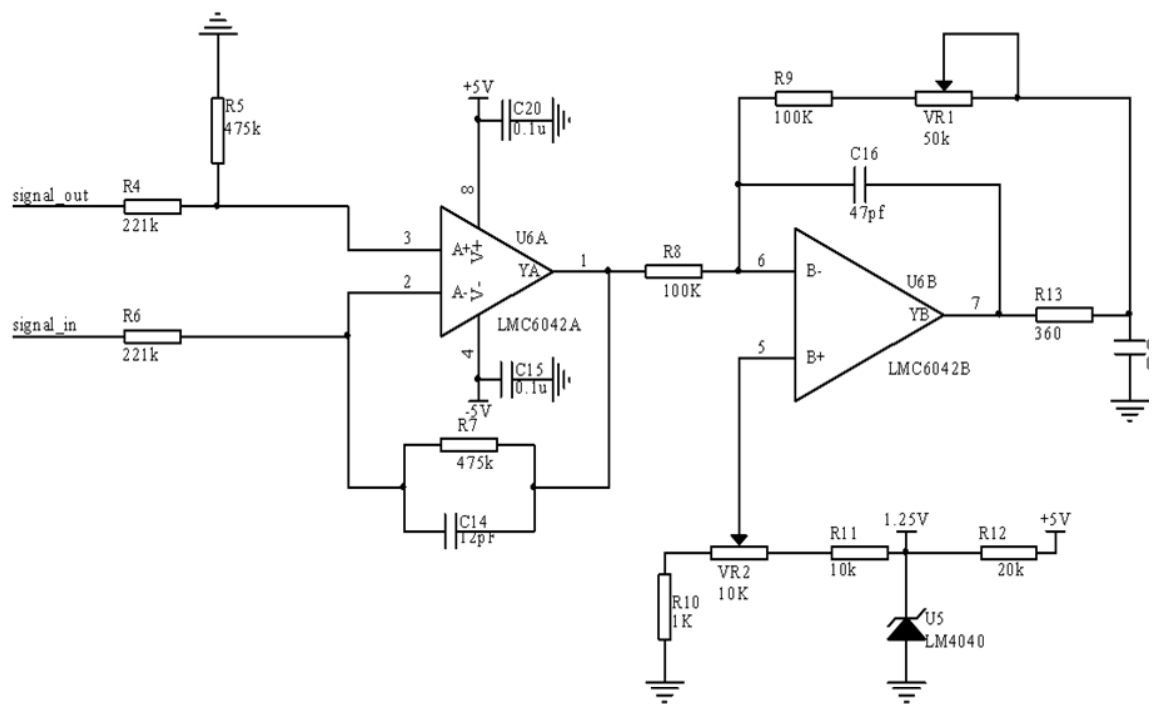

Fig. 2. Block diagram of signal processing circuit 
Observing that the first-stage used difference amplifier to perform differential operation, and the input sinusoidal signal was changed into DC signal, the function of the current-conveyor was to ensure the working probe is held at virtual ground when it was connected at the impedance. In addition, difference amplifier has the advantage of eliminating the Zero Drift. The second-stage amplifier was simply to give rise to the former-step's output signal, which aimed at improving stability of the testing system. The parameters of every component are as follows.

\section{System Architecture}

The system architecture is shown in the figure 3. The low-power MCU MSP430F149 is used as the core of the system acquisition and control, its power consumption and input leakage current (up to 50nA) is relatively low in the industry, the MSP430F149 is an industrial grade product, stable performance, high reliability. There are many work modes of the MSP430F149; the system power consumption is reduced by selecting a low power operating mode, so the MSP430F149 is especially suitable for handheld applications. It can reduce the number of external components and the cost of the instrument because of its 12-bit A / D converter. The keyboard and LCD modules for the operator provide a friendly human-machine interface; the real-time clock module can record the system running time; the memory module can save operating parameters and measurement data; the sensor interface is using a convenient PS / 2 interface.

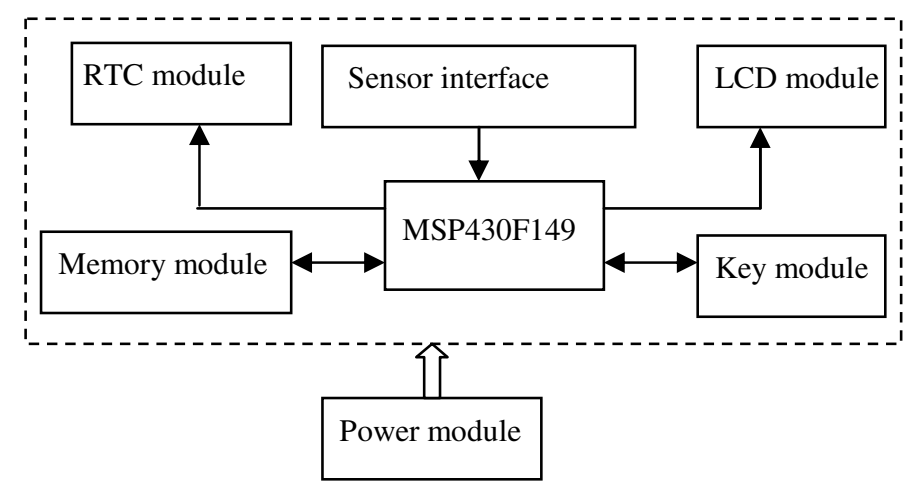

Fig. 3. The system architecture

\subsection{The Power Module}

The meter is using two $1.5 \mathrm{~V}$ batteries or a rechargeable battery, but the number of external components and the MSP430F149 must work at 3.3V, so it must increase the value of the power module. The circuit of the increasing voltage is shown in the figure 4. The chip of MAX1724, which character is a low quiescent current and high efficiency, is used in the power module. 


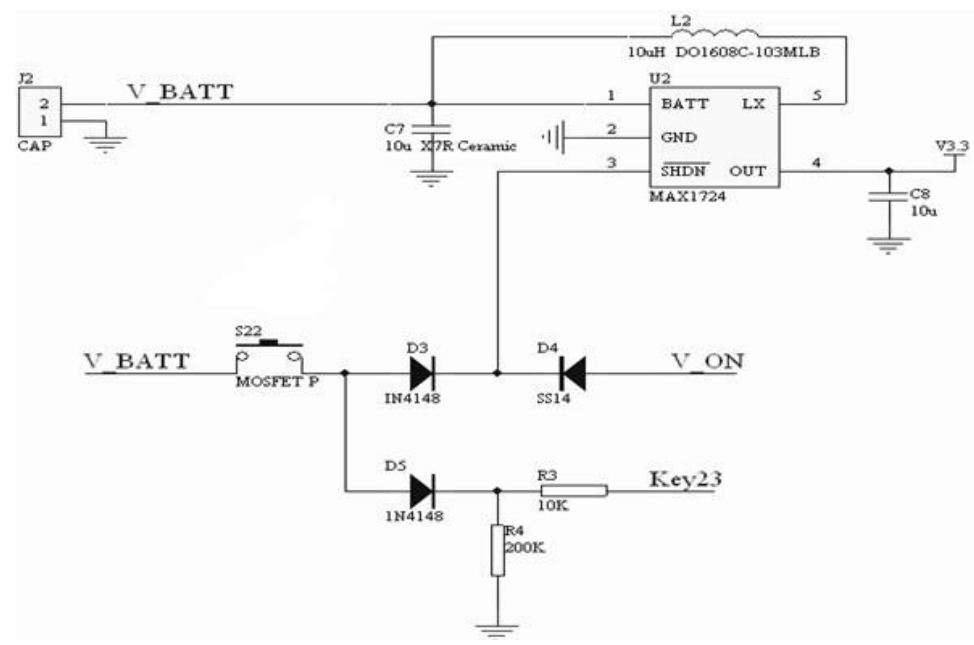

Fig. 4. The schematic of power

\subsection{The RTC Module}

The RTC module is designed for ensuring the system time synchronization, the schematic of the RTC is shown in the figure 5. The PCF8563 is a CMOS Real-Time Clock and calendar optimized for low power consumption. A programmable clock output, interrupt output, and voltage-low detector are also provided.

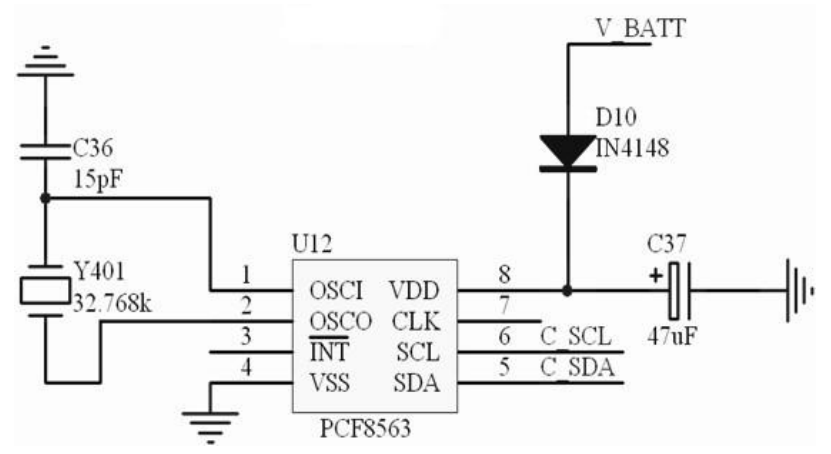

Fig. 5. The schematic of the RTC

\subsection{The Memory Module}

The 24LC02B is organized as one block of $256 \mathrm{X} 8$-bit memory with a 2-wire serial interface. Low-voltage design permits operation down to $2.5 \mathrm{~V}$, with standby and active currents of only $1 \mu \mathrm{A}$ and $1 \mathrm{~mA}$, respectively. its feature is low power consumption, simple external circuit, and write protection. The principle circuit of the 
memory module is shown in the figure 6 . The pins of $\mathrm{A} 0, \mathrm{~A} 1$, and $\mathrm{A} 2$ connect to the ground, its address is 000 , and the pin of the WP connects to the ground, so the chip can be all written. The pins of the SCL and the SDA connect respectively $10 \mathrm{~K}$ resistor, and make the level of the SCL and the SDA high, so can conveniently read and write.

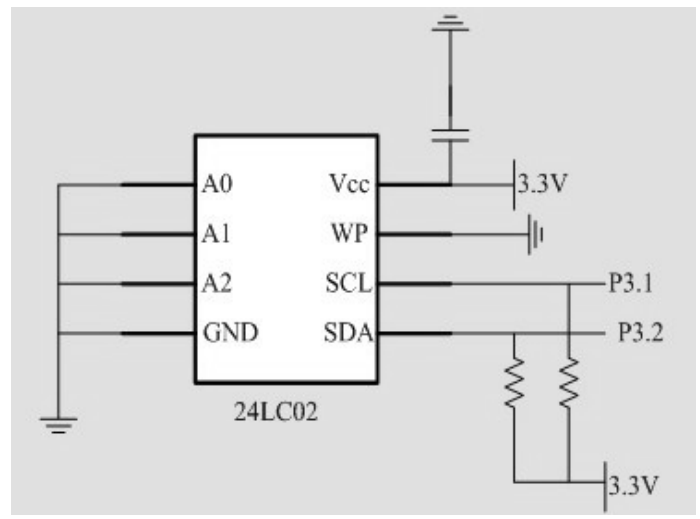

Fig. 6. The circuit of the memory module

\section{System Software Design}

The system software is modular in design, and they include a data measurement and processing module, a data storage module, a serial interface module, a keyboard and a LCD module, and act. The events are handled at the before platform and backstage. When the system is power on, initializes kinds of parameters, and then enters into the waiting stage; when receiving the measurement command, the system begins to measure, process, and storage the data.

\section{Experiment and Discussion}

\subsection{Experiment Analysis}

The test material was obtained from swine fillet and qualified by the quarantine system. The raw meat was fed to the meat grinder by inches to chop into fillet emulsion specimen, which were uniform in composition, within five times' wring. Using the electronic balance divided the emulsion into $10 \mathrm{~g}$ per portion by weight. Being intended to optimize the quality of test sample, delicately adding the demonized water into it until well-mixed was suggested. Afterwards, semi finished products were placed in the experimental beaker to prepare emulsion-aqueous solution of $10 \%$ and $20 \%$ respectively. 


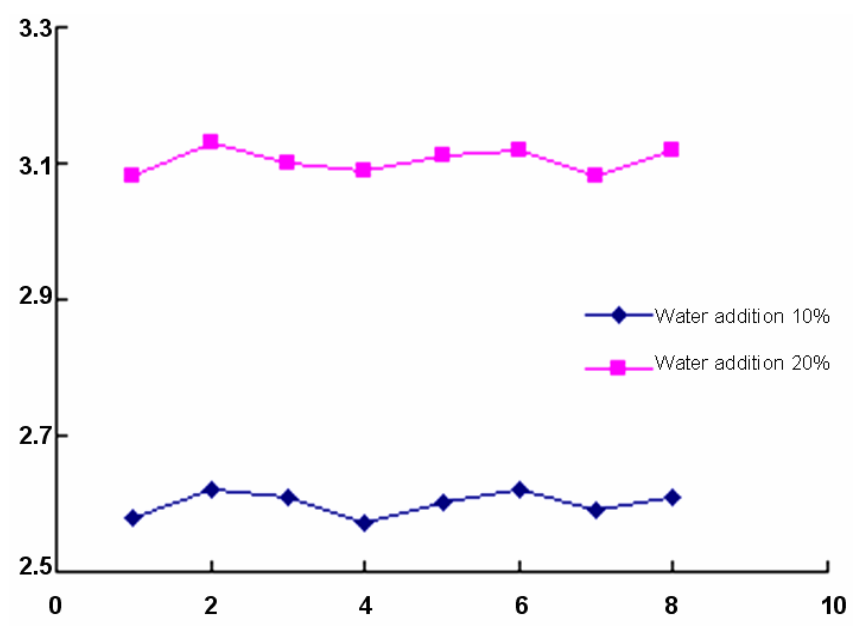

Fig. 7. Sensor output voltages within distinguished water-addition samples

The curvilinear was shown in Figure 7, the points of each group fluctuated around a certain value. With different water content added, the output results varied from one sample to another. Apparently, the more water it contained, the higher output voltage it may afterward lead to, and vice versa. Taking the $10 \%$-water-addition sample for an instance, we got the average of 3.10 and standard deviation of 0.01457 by calculating. The value was $0.47 \%$, which indicated a low dispersion degree. Similarly, that of the $20 \%$-water-addition sample was $0.49 \%$. So we dare to say, with the same testing sample, different sensors independently applied have obtained tolerably similar results, whose service performance and effectiveness can meet the need of measuring.

\subsection{Discussions}

Based on the research of the coaxial transmission line theory, the sensor and the meter for measuring pork quality were designed. The measurement results show that the stability and consistency of the sensor meet measurement requirements.

The plan of a low-power, battery-powered portable meter is designed. The characters of the MSP430F149 are low power consumption and real-time wake-up function, so the power consumption of the measuring meter of pork quality is rather lower, and this feature is ideal for portable meter. A friendly human-machine interface and standard PS/2 interface are very convenient for users.

In addition, the pork quality includes food quality, nutritional quality, technical quality, health quality and human quality, etc. At present, the quality of pork was inspected mainly through $\mathrm{PH}$ value, odor, color and the content of the water, the detection methods of using the $\mathrm{PH}$ value, odor, color were not in time in measuring the pork quality. The content of the water in the pork impacted on the pork quality was only considered in this paper; other parameters were ignored in this paper. If the pork quality was comprehensive responded, we must considered other factors, such as the $\mathrm{PH}$ value, odor, color, act. So the system of the pork quality evaluation is not put forward, and requires further study. 


\section{References}

1. Goldenberg, A.A., Lu, Z.: Automation of meat pork grading process. J. Computers and Electronics in Agriculture. 16(2), 125-135 (1997)

2. Ding, H., Duan, Y., He, T.: Spectral Research On The Effect of Optical Propa Gation in The Fat. J. Spectroscopy and Spectral Analysis 15(6), 19-24 (1995)

3. Ji, R., Wang, Z., Huang, L., et al.: Study of Measurement Methods for Detecting the Water Content in Deep Layer of Fresh Meat. J. Modern Scientific Instruments 1, 119-121 (2006)

4. Ding, Q., Wang, Z., Huang, L., et al.: Development of portable bio-impedance spectroscopy system for measuring porcine meat quality. J. Transactions of the CSAE 12(25), 138-144 (2009)

5. Zhang, L., Lyng, J.G., Brunton, N., et al.: Dielectric and thermo physical properties of meat batters over a temperature range of 5-85 ${ }^{\circ}$. J. Meat Science 68(2), 173-184 (2004)

6. Kent, M., Peymann, A., Gabriel, C., et al.: Determination of added water in pork products using microwave dielectric spectroscopy. J. Food Control 13(3), 143-149 (2002)

7. Zhang, L., Lyng, J.G., Brunton, N.P.: The effect of fat, water and salt on the thermal and dielectric properties of meat batter and its temperature following microwave or radio frequency heating. J. Journal of Food Engineering 80, 142-151 (2007) 